\title{
Descrição do uso do solo em duas propriedades rurais da localidade Cajá II, rodovia
}

\section{transassurini, Altamira-Pará}

\author{
Description of land use in two rural properties in Cajá II, transassurini highway, Altamira-Pará \\ Descripción de uso de suelo en dos propiedades rurales en Cajá II, carretera transassurini, \\ Altamira-Pará
}

Recebido: 31/10/2021 | Revisado: 08/11/2021 | Aceito: 13/01/2022 | Publicado: 15/01/2022

Ana Claudia Corrêa Moraes

ORCID: https://orcid.org/0000-0001-8651-822X

Secretaria de Estado de Meio Ambiente e Sustentabilidade, Brasil

E-mail: agro.semas@gmail.com

Andréia Silva da Luz

ORCID: https://orcid.org/0000-0002-7750-4245

Universidade Federal do Pará, Brasil

E-mail: andreialuz_18@hotmail.com

Roberta Rowsy Amorim de Castro

ORCID: https://orcid.org/0000-0003-3149-3170 Universidade Federal do Pará, Brasil E-mail: roberta_rowsy@hotmail.com

Francimary da Silva Carneiro

ORCID: https://orcid.org/0000-0002-1693-8779 Secretaria de Estado de Meio Ambiente e Sustentabilidade, Brasil

E-mail: francimarycarneiro@gmail.com

Simone Aparecida Almeida Araújo

ORCID: https://orcid.org/0000-0001-5402-0080 Instituto Federal de Educação Ciência e Tecnologia do Pará, Brasil E-mail: simonearaujo.vet@gmail.com

Lucyana Barros Santos

ORCID: https://orcid.org/0000-0001-9584-9274 Secretaria de Estado de Meio Ambiente e Sustentabilidade, Brasil

E-mail: lucyana_barros@ hotmail.com

Klewton Adriano Oliveira Pinheiro

ORCID: http://orcid.org/0000-0003-2696-4249 Instituto Federal de Educação, Ciência e Tecnologia do Pará, Brasil E-mail: klewton.pinheiro@gmail.com

Carla Vanessa Borges Castro

ORCID: https://orcid.org/0000-0002-5066-0862 Secretaria de Estado de Meio Ambiente e Sustentabilidade, Brasil E-mail: carlavbcastro@yahoo.com.br

Mayra Piloni Maestri

ORCID: https://orcid.org/0000-0002-8936-952X Secretaria de Estado de Meio Ambiente e Sustentabilidade, Brasil E-mail: mayrapmaestri@ hotmail.com

Ana Paula Magno do Amaral

ORCID: https://orcid.org/0000-0002-1749-791X Secretaria de Estado de Meio Ambiente e Sustentabilidade, Brasil E-mail: magno_ana@yahoo.com.br

\begin{abstract}
Resumo
Este estudo foi realizado em duas propriedades localizadas no Travessão do Cajá II, rodovia Transassurini, no mês de agosto de 2007, com o objetivo de caracterizar os diferentes usos do solo. As famílias realizam implantações de culturas anuais, como: arroz, feijão, milho e mandioca. Os proprietários utilizam para o preparo do solo o sistema itinerante. Observa-se nas duas propriedades, que os agricultores usam o mesmo método para o plantio de culturas perenes e para manejo de pastagem, onde na propriedade I é empregado o sistema de roça, no qual o proprietário prioriza a preservação do solo e seus nutrientes. A propriedade II apresenta preservação de matas ciliares, mantendo assim os igarapés em bom estado. A propriedade I dispõe de 20 ha. de mata virgem, enquanto a propriedade da família II preserva aproximadamente 73 ha. Em ambos os lotes os solos das áreas de pastagens apresentam-se compactados, devido ao pisoteio freqüente dos bovinos.
\end{abstract}

Palavras-chave: Plantações; Culturas; Agricultura; Pastagens; Mata Ciliar. 


\begin{abstract}
The survey was conducted in two properties located in the Cajá Citation II, Transassurini highway, in the month of August in the year 2006, during the first stage of the class of Agronomy 2006, aiming to characterize the different uses of land in these establishments. The families held deployments of annual crops such as rice, beans, maize and cassava. The owners use to prepare the ground the itinerant system. Notes on the two properties, that farmers use the same method to the planting of perennial crops and grassland management, the family I employ the system of grazing, as the owner prioritizes the preservation of soil and its nutrients. The ownership of the family II presents gallery preservation of forests, thereby maintaining the igarapés in good condition. The ownership of the family I has 20 hectares of virgin forest, while the ownership of the family II preserves approximately 73 hectares. In both the land lots of areas of grassland have to be compressed, due to frequent trampling of cattle.
\end{abstract}

Keywords: Plantations; Cultures; Agriculture; Pastures; Riparian Forest.

\title{
Resumen
}

Este texto es el realizada en dos predios ubicados en Travessão do Cajá II, Carretera Transassurini, en agosto de 2007, con el objetivo de caracterizar los diferentes usos del suelo. Las familias implementan cultivos anuales, tales como: arroz, frijol, maíz y mandioca. Los propietarios utilizan el sistema itinerante para la preparación del suelo. Se observa en ambas propiedades, que los agricultores utilizan el mismo método para la siembra de cultivos perennes y para el manejo de pastos, donde en la propiedad se utiliza el sistema de quema, en el que el propietario prioriza la preservación del suelo y sus nutrientes. La propiedad II presenta la preservación de los bosques ribereños, manteniendo los arroyos en buen estado. Propiedad tengo 20 ha. de bosque virgen, mientras que la propiedad de la familia II conserva aproximadamente 73 ha. En ambos lotes, los suelos de las áreas de pastoreo están compactados, debido al frecuente pisoteo del ganado.

Palabras clave: Plantaciones; Cultivos; Agricultura; Pastos; Bosque de Ribera.

\section{Introdução}

A agricultura é uma atividade antrópica essencial para toda e qualquer sociedade, independente do nível de desenvolvimento. A grande questão contemporânea é saber como mantê-la produtiva sem afetar os diferentes ecossistemas terrestres. (Gualberto et al 2003, Botelho et al 2020, Rodrigues et al 2020).

O solo pode ser considerado a base de sustentação dos sistemas agrícolas (Reichert et al 2003, Serra et al 2020). Assim, perdas nas propriedades, que reduzam a capacidade de sustentar o crescimento vegetal ou que implique riscos ambientais, causam impacto negativo de grande significação para as comunidades rurais, com repercussões no meio urbano (Batista et al 2020, Costa \& Castigo 2021). Por outro lado, a melhoria do ambiente edáfico tem efeitos positivos sobre todo o ambiente, revestindo de grande importância o conhecimento da qualidade do solo e sua quantificação via indicadores físicos, químicos e biológicos (Reichert et al apud Serra et al, 2007, Silva et al 2021).

Com base nestas informações este estudo parte da observação em lócus (in locu) da utilização do solo em duas propriedades agrícolas, situadas na comunidade do Cajá II, Rodovia Transassurini, município de Altamira, estado do Pará e parte do pressuposto de que os agricultores não detêm de saberes técnicos no que se refere à utilização de áreas para a implantação de pastagens e culturas, ou seja, que eles realizam este manejo de forma desordenada, sem preocupação com a preservação ambiental, não obedecendo a critérios técnicos. Nesta perspectiva, a pesquisa de campo se desenvolveu através de observação direta na unidade de produção, entrevistas e caminhadas realizadas em companhia dos agricultores nas áreas relevantes, objetivando caracterizar e descrever os diferentes usos do solo, assim como os sistemas de preparo deste, adotados pelos agricultores em seus lotes.

\section{Metodologia}

A pesquisa foi realizada em duas propriedades agrícolas, localizadas na Rodovia Transassurini, comunidade do cajá II, no município de Altamira, Pará, pertencentes aos Srs. Antonio Olegário da Silva e Guilherme Coelho da Silva, propriedade I e propriedade II, respectivamente.

Os levantamentos foram obtidos por meio de observações das diferentes utilizações do solo, entrevistas feitas aos 
proprietários, objetivando adquirir informações sobre o manejo e as divisões das parcelas, assim como caminhadas realizadas nas propriedades em companhia dos agricultores, ocorridas no período de 09 a 16 de agosto de 2007.

\section{Resultados}

A área da propriedade I mede em torno de 65 ha, onde já foram explorados 45 ha. Destes, 24 ha são usados no cultivo de pastagens, 19 ha para os cultivos de cacau (Theobroma cacao L.), cupuaçu (Theobroma grandiflorum (Willd. ex. Spreng.) Schum.) e café (Coffea ssp). Existe ainda, um espaço utilizado pela família para plantação de milho (Zea mays L.) consorciado com feijão (Phaseolus vulgaris L.) e mandioca (Manihot esculenta Crantz.), implantado todos os anos para o consumo da família, no caso da mandioca, ela é usada para a fabricação da farinha, não só para consumo próprio, como também para venda e troca em produtos como açúcar e café.

No que se refere ao preparo da área para o plantio, foi observado o uso do modelo tradicional de preparo do solo, no qual é utilizado o fogo precedido por broca e derruba. Em seguida, planta-se a cultura, que pode ser anual ou perene. De acordo com o tipo de solo, o mesmo pode servir para cultivo de pastagem.

Na propriedade I, não é utilizado o fogo para a limpeza das pastagens. Neste caso, é empregado o sistema de roça, que prioriza a conservação do solo e seus nutrientes (Almeida et al 2006, Alves et al 2013, Almeida 2019). Este processo é feito duas vezes por ano, com a utilização de ferramentas como foice e facão.

A propriedade II é constituída por dois lotes confrontantes, estabelecendo um total de aproximadamente 140 ha, destes 67 ha foram explorados, onde a mata nativa que ocupava 100\% da área no ano de 1986, quando o agricultor chegou à propriedade, atualmente ocupa 72,63 ha. (LUZ, A. S. et al. 2006. 56 p.).

Do total explorado destaca-se 39,3 ha para a implantação de pastagens, local este em que o solo apresenta-se bastante compactado, devido ao pisoteio constante de bovinos. Existem também espaços de capoeiras com idades entre um e 10 anos, em um total de 3,26 ha.

Aproximadamente oito ha são reservados para a implantação de culturas anuais, como o arroz (Oriza sativa L.), feijão (Phaseolus vulgaris L.), milho (Zea mays L.) e mandioca (Manihot esculenta Crantz.).

Além dos agroecossistemas mencionados, a propriedade conta ainda com áreas onde são implantadas culturas perenes, sendo 8 ha para o cacau (Theobroma cacao L.), com idades de 3, 4, 10 e 20 anos, 6,96 ha para a pimenta-do-reino (Piper nigrun L.) e 0,33 ha para o plantio de café (Coffea ssp).

É utilizado pela família o sistema de agricultura itinerante, que consiste na derruba da mata, em seguida queima-se e implanta-se a cultura ou pastagem, porém, o agricultor preocupa-se com o sistema adotado, por este ser nocivo ao meio ambiente, todavia continua a utilizá-lo por considerá-lo mais econômico. Os agricultores persistem nesse sistema, por ser de baixo custo e de fácil adoção, em função da baixa fertilidade da maioria dos solos da região amazônica, do elevado custo de fertilizantes e corretivos (Rego \& Kato 2018, Alves \& Modesto Junior 2020, Silva et al 2021, Soares et al 2021).

Há grande diversidade vegetal, fato constatado pela presença de árvores de lei, plantas medicinais e ornamentais. No perímetro da casa do agricultor, há árvores frutíferas e uma horta que supre as necessidades domésticas da família.

Nas plantações de cacau, o solo apresenta-se com quantidade considerável de matéria orgânica devido à decomposição da liteira, assim como na área da mata primária e do açaí nativo (Euterpe oleracea Mart.) onde o solo encontrase úmido, apresentando coloração bastante escura. Próximo a esta área de açaí, que equivale a um ha encontra-se a mata ciliar que foi mantida pelo agricultor e em consequência os igarapés encontram-se também em bom estado de preservação. Para a disponibilidade e qualidade de água, é necessário a conservação das faixas de vegetação nativa que margeiam seus corpos d’água, denominadas de matas ciliares (Santos et al 2021, Cantuária et al 2021).

A propriedade apresenta boa disponibilidade hídrica, com um total de cinco igarapés cortando os lotes, estes são 
utilizados para fornecer água aos bovinos e outros animais, para a irrigação de dois viveiros de cacau e esporadicamente para executar tarefas domésticas como lavagem de roupas.

\section{Conclusão}

Pode-se constatar com essa pesquisa que as famílias estudadas estão interferindo nas características originais do solo, visto que utilizam sistemas de manejo, que possibilitam grandes mudanças relacionadas à aptidão agrícola destes. Neste sentido, ambos os proprietários utilizam-se de fogo no preparo da área para implantação de culturas.

Na propriedade I, os solos já foram muito explorados, porém o agricultor preocupa-se com as consequências que o manejo da preparação do solo pode acarretar, fato evidenciado pela não utilização do fogo para limpeza das pastagens, no intuito de conservar o solo.

Na propriedade II, as áreas de pastagens já foram bastante cultivadas, e por este motivo encontram-se bastante degradadas pelo pisoteio dos bovinos. Nas plantações de cacau existem quantidades consideráveis de matéria orgânica, o que ajuda na manutenção e conservação do solo nestas áreas. Além disso, o agricultor mantém as matas ciliares, no intuito de preservar os igarapés.

\section{Referências}

Almeida, E., Sabogal, C. \& Brienza Júnior, S. (2006). Recuperação de Áreas Alteradas na Amazônia Brasileira: Experiências Locais, Lições Aprendidas e Implicações para Políticas Públicas. Belém, Cifor. http://www.alice.cnptia.embrapa.br/alice/handle/doc/375969.

Almeida, L. N. (2019). Queimadas agrícolas geram danos ao solo e à produção. Ministério da Agricultura Pecuária e Abastecimento -MAPA. Tribuna do Norte. www.tribunadonorte.com.br/noticia/queimadas-agricicolas-geram-danos.

Alves, R. N., Junior, M., \& Souza, M. D. (2013). Roça sem fogo planejamento da mandioca. Revista e Cidadania \& Meio Ambiente.

Alves, R., \& Modesto Junior, M. D. S. (2020). Roça sem fogo: da tradição das queimadas à agricultura sustentável na Amazônia. Embrapa Amazônia Oriental-Livro técnico (INFOTECA-E). http://www.infoteca.cnptia.embrapa.br/infoteca/handle/doc/1119432

Batista, J. A. Dos S., Barbosa, M. De S., Silva, F. N. L. Da, Oliveira, L. C. De, \& Sousa, R. L. De. Descrição social, econômico e ambiental de comunidades rurais no Acará, Pará, Brasil. Research, Society and Development, 9(7), e423974294, 10.33448/rsd-v9i7.4294.

Botelho, M. G. L., Pimentel, B. Dos S., Furtado, L. G., Lima, M. Do C. S., Carneiro, C. R. De O., Batista, V. De A., Marinho, J. L. M., Monteiro, A. L. P. R., Silva, T. P. Da, Pontes, A. N., \& Costa, M. Do S. S. (2020). Agrotóxicos na agricultura: agentes de danos ambientais e a busca pela agricultura sustentável. Research, Society and Development, 9(8), e396985806, 10.33448/rsd-v9i8.5806.

Cantuária, P. De C., Medeiros, T. D. S., Sobrinho, M. S., Silva, R. B. L. E, Costa Neto, S. V. Da, Araújo, C. B. De., Furtado, M. F. De M., Krahl, A. H., Soares, A. C. S., Farias, A. L. F., Potyguara, P. M. B., Almeida, S. S. M. Da S. De., \& Farias, J. E. Dos S. (2021). Riqueza Vegetal da Bacia Hidrográfica do Igarapé da Fortaleza, AP-010, Amapá, Brasil. Research, Society and Development, 10(13), e503101321290.

Costa, J. I. M. \& Da. Castigo, P. (2021) Análise dos progressos, constrangimentos e desafios do programa agrário "Sustenta" em Moçambique. Research, Society and Development, 10(14), e244101421682. 10.33448/rsd-v10i14.21682.

Gualberto, V., Mello, C. R., \& Nóbrega, J. C. A. (2003). O Uso do Solo no Contexto Agroecológico: Uma Pausa para Reflexão. Revista Informe Agropecuário, 24(220), 18-28.

Luz, A. S. Da, Guimarães, J. De S., Castro, R. R. A. \& De Pissinatti, V. (2006). Relatório do I-Estágio de Campo realizado nas propriedades dos Srs. Guilherme Coelho da Silva e Francisco de Assis Coelho da Silva, localizado na comunidade do Cajá II, no km 40, Rodovia Transassurini, Município de Altamira - PA. Altamira: UFPA. 56 p.

Moraes, A. C. C., Nicareta, E. J., \& França, H. B. (2006). Relatório do I Estágio de Campo realizado nas Propriedades dos Srs.Antonio Olegário da Silva e Valdelice Brito Silva, localizadas no travessão do cajá II, no km 40, Rodovia Transassurini, Município de Altamira - PA. Altamira: UFPA. 53 p.

Rego, A. K. C., \& Kato, O. R. (2018). Agricultura de corte e queima e alternativas agroecológicas na Amazônia. Novos Cadernos NAEA, Belém, 20 (3), ISSN 2179-7536. http://dx.doi.org/10.5801/ncn.v20i3.3482

Reichert, J. M. \& Reinert, D. \& Braida, J. (2003). Qualidade dos solos e sustentabilidade de sistemas agrícolas. Ciência \& Ambiente. 27.29.

Reichert, J. M., Reinert, D. J., \& Braida, J. A. (1990). Qualidade dos Solos e Sustentabilidade de sistemas agrícolas. Revista Ciência e Ambiente. Universidade Federal de Santa Maria,.1(1).

Rodrigues, C. De O., Lopes, M. L. B., Rebello, F. K., \& Corrêa, R. Do S. Da S. (2020). Caracterização socioeconômica dos sistemas de Agricultura Urbana e Periurbana adotados no Município de Irituia, Pará, Brasil. Research, Society and Development, 9(7), e795974764, 10.33448/rsd-v9i7.4764. 
Research, Society and Development, v. 11, n. 1, e55211122581, 2022

(CC BY 4.0) | ISSN 2525-3409 | DOI: http://dx.doi.org/10.33448/rsd-v11i1.22581

Santos, D. M. D. Dos., Silva, M. F. Da, \& Lima, P. A. F. (2021). Caracterização do Igarapé Chico Reis, Rorainópolis - RR e restauração de matas ciliares na Amazônia: um referencial teórico. Research, Society and Development, 10(15), e341101522816. 10.33448/rsd-v10i15.22816.

Serra, A. B. et al, (2007). Estoque de Serrapilheira e matéria orgânica do solo em sistemas alternativos ao uso do fogo, desenvolvido por agricultores familiares na região Transamazônica e Xingu - Oeste do Pará. In: Anais da $7^{a}$ Semana de Integração das Ciências Agrárias. Altamira, PA: UFPA.

Serra, A. B., Carvalho, C. J. R., Alves Júnior, M., \& Sá, T. D. A. (2020). Fertilidade dos solos em cultivo de cacau em roça sem queimar e cultivo de cacau com uso do fogo. Atena editora, 33p. 10.22533/at.ed.387200402

Silva, A. O. Da., Silva, A. O. Da, Santos, D. C. R., Rosário, I. C. B. Do., Barata, H. Da S., \& Raiol, L. L. (2021). Da tradição a técnica: perspectivas e realidades da agricultura de derruba e queima na Amazônia. Research, Society and Development, 10(1), e38310111799. 10.33448/rsd-v10i1.11799.

Silva, H. S., Chaves, J. Da S., Nascimento, J. P. S. Do., Matos, S. M. De., Brito Neto, A. F. De., Leite, J. L., Pereira, H. R., \& Brito, W. A. De. (2021). Atividade microbiana no solo em sistema de produção consorciado. Research, Society and Development, 10(14), e534101422366, 10.33448/rsd-v10i14.22366.

Soares, A. C. S., Silva, M. S. Da., Amaral, S. Da S., Queiroz, L. O. De., Pereira, A. R. Dos S., Abreu, V. S., Costa Neto, W. V. Da., Rabelo, F. G., Araújo, N. M., \& Cantuária, P. De C. (2021). Queimar ou não queimar? Um estudo de caso da roça sem queima na comunidade São Sebastião do Livramento-Cutias/AP. Research, Society and Development, 10(16), e155101622537. 10.33448/rsd-v10i16.22537. 